\title{
Self-reported needs of patients with Parkinson's disease during COVID-19 emergency in Italy
}

\author{
Tommaso Schirinzi $^{1}$ (D) Rocco Cerroni ${ }^{1}$ - Giulia Di Lazzaro ${ }^{1}$. Claudio Liguori ${ }^{1}$ - Simona Scalise ${ }^{1} \cdot$ Roberta Bovenzi $^{1}$. \\ Matteo Conti ${ }^{1}$ - Elena Garasto ${ }^{1}$ • Nicola Biagio Mercuri ${ }^{1,2}$ • Mariangela Pierantozzi ${ }^{1}$ • Antonio Pisani ${ }^{1,2}$. \\ Alessandro Stefani ${ }^{1}$
}

Received: 11 April 2020 / Accepted: 25 April 2020 / Published online: 3 May 2020

(C) Fondazione Società Italiana di Neurologia 2020

\begin{abstract}
Because of COVID-19 outbreak, regular clinical services for Parkinson's disease (PD) patients have been suddenly suspended, causing worries, confusion and unexpected needs in such frail population. Here, we reviewed the messages spontaneously sent by patients to an Italian PD clinic during the first two weeks of COVID-19 lockdown (9-21 March 2020), in order to highlight their main needs and then outline appropriate strategies of care for this critical period. One hundred sixty-two messages were analysed. Forty-six percent queried about clinical services; $28 \%$ communicated an acute clinical worsening for which a therapeutic change was done in $52 \%$ of cases; $17 \%$ (those patients with younger age and milder disease) asked about the relationship between PD and COVID-19; $8 \%$ informed about an intercurrent event. Our analysis suggests that PD patients' needs during COVID-19 emergency include appropriate and complete information, a timely update on changes in clinical services, and the continuity of care, even in a remote mode. By addressing these issues, acute clinical worsening, complications and subsequent therapeutic changes could be prevented. In this perspective, telecommunication systems and virtual medicine should be implemented.
\end{abstract}

Keywords COVID-19 $\cdot$ Parkinson's disease $\cdot$ Coronavirus $\cdot$ Neurological diseases $\cdot$ Telemedicine

\section{Introduction}

Italy was hardly scourged by Coronavirus disease 2019 (COVID-19) pandemia [1] and, on 9 March 2020, extraordinary measures (lockdown) have been ordered. Hospitals' routine activities were stopped [1] and a huge number of neurologists were employed to treat COVID-19 patients. Therefore, follow-up visits and scheduled activities for patients with Parkinson's disease (PD) were suddenly suspended.

Media provided continuous information on facts related to COVID-19 outbreak; however, sometimes, it turns out to be incomplete or inappropriate [2]. The lack of adequate information, together with the emergency's spread and the regular

Tommaso Schirinzi

t.schirinzi@yahoo.com

1 Department of Systems Medicine, University of Roma Tor Vergata, Via Montpellier, 00133 Rome, Italy

2 IRCCS Fondazione Santa Lucia, Rome, Italy clinical services' suspension, caused worries and confusion in PD patients, which massively contacted their doctors for several questions.

In this study, we collected the major queries raised by patients from a PD clinic of a single Italian centre over the first two weeks of COVID-19 lockdown. We aimed to focus their main needs and identify opportune strategies to adequately assist PD patients during this emergency.

\section{Methods}

E-mails, phone texts and phone vocal messages spontaneously sent form PD patients or caregivers to the PD Clinic of Tor Vergata University Hospital (Rome, Italy) from 9 to 21 March 2020 were collected and analysed.

Queries and communications (Q) were classified in 4 groups depending on the content: (Q1) relationship between COVID-19 and PD; (Q2) acute changes in neurological symptoms; (Q3) occurrence of intercurrent medical/surgical conditions; (Q4) clinical services. For each patient, age, sex, disease 
duration, Hoehn and Yahr (HY) stage and address were collected retrospectively from the electronic chart.

Possible therapeutic modifications due to Q2 were recorded. The study followed local ethical standards and the principles of Helsinki declarations.

\section{Statistical analysis}

Distribution of continuous variables was assessed and nonnormally distributed ones were $\log 10$ transformed to allow statistical analysis. Descriptive statistic was calculated for categorical variables. Group differences were analysed by parametric or non-parametric tests, as appropriate (Software: IBMSPSS).

\section{Results}

One hundred sixty-two communications were received, 89.2\% from Rome area, 8.6\% from South-Italy, and 2.2\% from other northern-centre regions. Figure 1 a represents prevalence of queries. Q4 (about clinical services) was the most frequent $(46 \%)$. Table 1 summarizes clinical-demographic parameters of patients. Age significantly differed $(F(3,126)=$ $3.35 ; p=0.02)$, being lower for Q1 (55.3 years) compared with Q4 (65.3) (Bonferroni post hoc, $p=0.01$ ). As well, HY significantly differed $(F(3,117)=4.9 ; p=0.003)$, being higher in Q3 (3.8) compared with Q1 (2.1) $(p=0.002)$.

Forty-six patients referred acute clinical changes during the lockdown (Q2). Among these, 50\% experienced an increase in motor disturbances (tremor, rigidity, gait difficulties); $25 \%$ had augmented anxiety and $18 \%$ developed/worsened neuropsychiatric symptoms (NPS, e.g. hallucinations, agitation, psychosis); $16 \%$ complained about other non-motor symptoms (NMS, e.g. fatigue, pain) (Fig. 1b). In $52.3 \%$ of Q2 patients, doctors provided therapeutic changes (in remote, according to standard clinical practice): in $33.3 \%$ of cases, the dopaminergic therapy was modified; in $41.7 \%$, an anxiolytic drug (benzodiazepine) was introduced; in $25 \%$, a neuroleptic was introduced/up-titrated.

Q3 patients (those with higher HY stage) communicated an intercurrent medical/surgical event, which required in $46.2 \%$ of cases a hospital admission (1 decease). COVID-19 infection was suspected in $23.1 \%$ of them but confirmed in none.

Q4 represented the most numerous group. Patients asked about confirmation of scheduled activities (e.g. visits, trials) in $47 \%$ of cases, required prescriptions for drugs or certifications in $57 \%$ and complained about the suspension of rehabilitation programmes (physiotherapy, PT) in 13\% (Fig. 1c).

\section{Discussion}

We reviewed all queries and communications spontaneously provided by PD patients to an Italian PD clinic team during the first two weeks of the COVID-19 lockdown, in order to highlight their main needs and develop opportune strategies of assistance during this critical period.

In $46 \%$ of cases, patients contacted for issues regarding clinical services, namely to be updated about scheduled
Fig. 1 a Queries prevalence of PD patients. (Q1) Relationships between COVID-19 and PD; (Q2) changes in neurological symptoms occurred in that period; (Q3) occurrence of intercurrent medical/surgical conditions in that period; (Q4) clinical services. $\mathbf{b}$ and $\mathbf{c}$ Frequency of various items pertinent to Q2 and Q4. b Frequency of symptoms worsened during the lockdown. c Frequency of issues regarding clinical services interrupted by the lockdown. d. disturbances, other abbreviations spelled out in the text
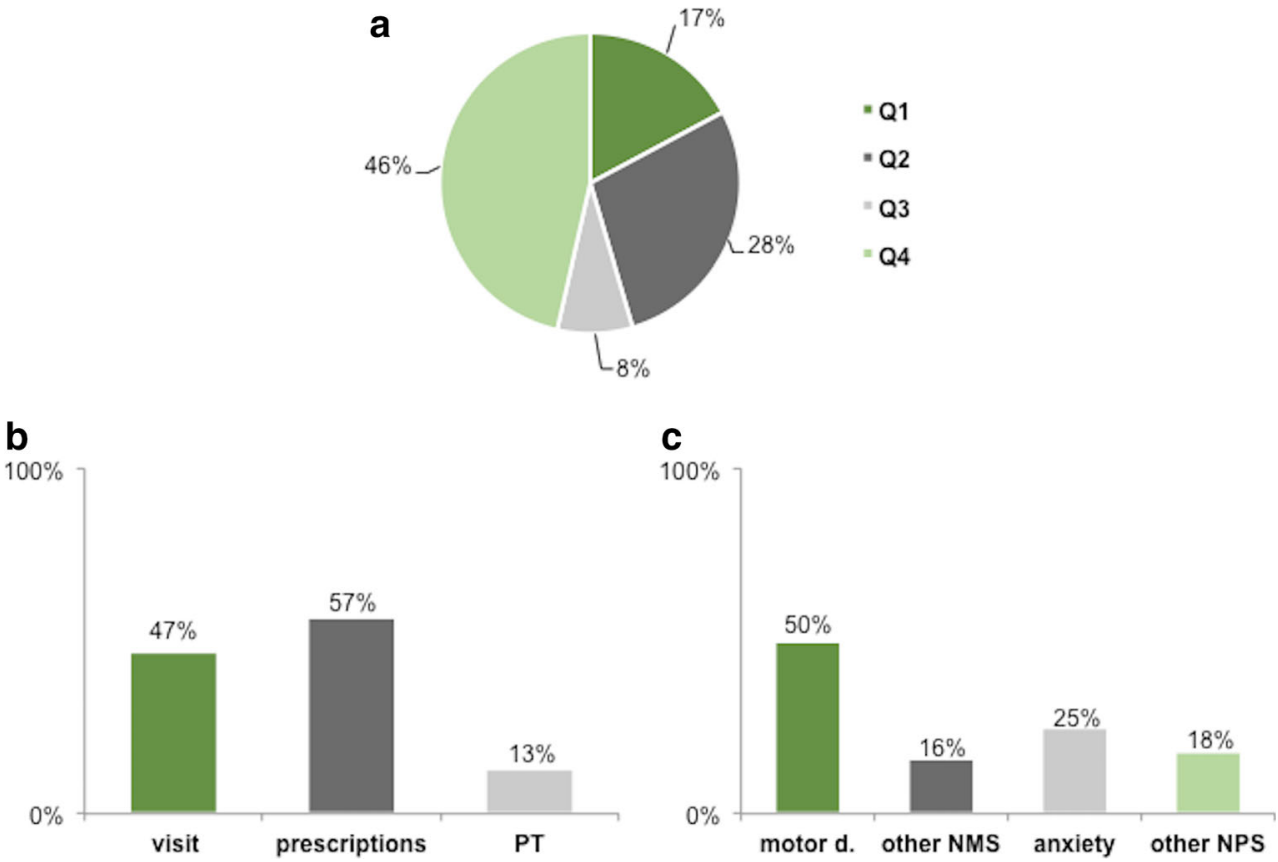
Table 1 Demographic and clinical data of the study population

\begin{tabular}{clllll}
\hline Group & $N$ & Sex, F\%/M\% & Age (y), mean (SD) & Duration (y), mean (SD) & HY, mean (SD) \\
\hline All & 162 & $46 \% / 54 \%$ & $64.9(13.1)$ & $8.9(5.6)$ & $2.5(0.9)$ \\
Q1 & 28 & $53 \% / 47 \%$ & $55.3(12.3)$ & $6.9(4.4)$ & $2.1(0.9)$ \\
Q2 & 46 & $54 \% / 46 \%$ & $62.7(16.4)$ & $8.9(5.6)$ & $2.5(0.9)$ \\
Q3 & 13 & $46 \% / 54 \%$ & $62.2(14.4)$ & $13.7(7.5)$ & $3.8(0.9)$ \\
Q4 & 75 & $40 \% / 60 \%$ & $65.3(11.9)$ & $9.1(5.5)$ & $2.5(0.9)$ \\
\hline
\end{tabular}

$N$, number; $y$, years; $F / M$, female/male. Other abbreviations spelled out in the text activities (visits, trials), to ask for prescriptions or to complain about the suspension of PT.

Twenty-eight percent of patients communicated an acute clinical worsening, in either motor disturbances or neuropsychiatric and other non-motor symptoms. Although none of these patients was affected by COVID-19, they experienced the worsening of their condition at the same time of the lockdown and the infection outbreak, consistently with the wellknown detrimental effect of stress on PD symptomatology [3]. Fifty-two percent of these patients required therapeutic changes, often consisting of a higher use of benzodiazepines and neuroleptics, besides the adjustment of dopaminergic drugs.

Seventeen percent of patients, specifically those younger, with a milder disease, contacted us to ask for clarification on the relationships between COVID-19 and PD, in particular on the risk of contracting the infection because of PD and its medications.

Finally, $8 \%$ of patients, those more severely affected, communicated the appearance of an intercurrent medical/surgical event. COVID-19 disease was suspected but not confirmed in 3 of these patients.

This study has several limitations. First, this is not a systematic structured survey on a PD population but a collection of self-reported communications, spontaneously provided by patients through e-mails, phone texts and vocal messages. Actually, needs from older, cognitively impaired and uneducated, or more severely affected patients could have been underestimated. Then, data came from the area of Rome, which was less affected by COVID-19 than other regions, limiting the generalization to the whole Italian population. Finally, we focussed on the beginning of the lockdown and the early phases of the COVID-19 emergency.

Nevertheless, our analysis suggests that the main PD patients' needs in this emergency were two. First, the correct and timely information. Indeed, an accurate communication with patients on the relations between PD and COVID-19 and an opportune update on the clinical services' schedule might temper their stress and limit possible symptoms worsening, responsible, in turn, for unexpected therapeutic interventions. Second, the continuity of care, even in a "smart" mode. PD patients indeed require continuous treatments and regular checks. Accordingly, the boost of telemedicine and "virtual" visits or PT could be strikingly helpful, as well as the digitalization of different services.

Web-based technologies and virtual telecommunication, although hindered by several limitations (the possible technical issues for older and advanced patients or privacy/ bureaucracy concerns) $[4,5]$, thus represent the way to ensure the due care to PD patients during COVID-19 emergency.

Data availability Data are available on request.

\section{Compliance with ethical standards}

Conflict of interest The authors declare that they have no conflict of interest.

Ethical approval None.

\section{References}

1. Remuzzi A, Remuzzi G (2019) Health policy COVID-19 and Italy: what next? https://doi.org/10.1016/S0140-6736(20)30627-9

2. Ippolito G, Hui DS, Ntoumi F, Maeurer M, Zumla A (2020) Toning down the 2019-nCoV media hype - and restoring hope. Lancet Respir Med 8:230-231

3. Hemmerle AM, Herman JP, Seroogy KB (2012) Stress, depression and Parkinson's disease. Exp Neurol 233:79-86

4. Dorsey ER, Achey MA, Beck CA, Beran DB, Biglan KM, Boyd CM, Schmidt PN, Simone R, Willis AW, Galifianakis NB, Katz M, Tanner CM, Dodenhoff K, Ziman N, Aldred J, Carter J, JimenezShahed J, Hunter C, Spindler M, Mari Z, Morgan JC, McLane D, Hickey P, Gauger L, Richard IH, Bull MT, Mejia NI, Bwala G, Nance M, Shih L, Anderson L, Singer C, Zadikoff C, Okon N, Feigin A, Ayan J, Vaughan C, Pahwa R, Cooper J, Webb S, Dhall R, Hassan A, Weis D, DeMello S, Riggare SS, Wicks P, Smith J, Keenan HT, Korn R, Schwarz H, Sharma S, Stevenson EA, Zhu W (2016) National randomized controlled trial of virtual house calls for people with Parkinson's disease: interest and barriers. Telemed eHealth 22:590-598. https://doi.org/10.1089/tmj.2015.0191

5. Marxreiter F, Buttler U, Gassner H, Gandor F, Gladow T, Eskofier B, Winkler J, Ebersbach G, Klucken J (2019) The use of digital technology and media in German Parkinson's disease patients. J Park Dis 10:717-727. https://doi.org/10.3233/JPD-191698

Publisher's note Springer Nature remains neutral with regard to jurisdictional claims in published maps and institutional affiliations. 